\title{
Qualidade da Relação Líder-Membro: Evidências de Validade da Escala Multidimensional
}

\author{
Marcela Guimarães Côrtes ${ }^{1}$ \\ Maira Gabriela Santos de Souza ${ }^{2}$ \\ Katia Elizabeth Puente-Palacios ${ }^{1}$ \\ ${ }^{1}$ Universidade de Brasília, Brasília, DF \\ ${ }^{2}$ Empresa Brasileira de Pesquisa Agropecuária, Brasilia, DF
}

\begin{abstract}
Resumo
Este artigo objetivou identificar evidências de validade da estrutura interna da versão brasileira da escala multidimensional da qualidade da relação líder-membro (LMX-MDM). Aplicou-se a LMX-MDM a 363 pessoas. Realizou-se análise fatorial exploratória e confirmatória, e análise da correlação com outros construtos. A escala apresentou nove itens distribuídos em três fatores de primeira ordem que se agrupam em um fator global: Respeito Profissional $(\alpha=0,93)$, Afeto $(\alpha=0,92)$ e Lealdade $(\alpha=0,90)$, explicando $69,39 \%$ da variância do fenômeno. Os indicadores de ajuste foram satisfatórios $\left(\chi^{2} / g\right.$.l. $=2,20 ; G F I=0,97 ; C F I=0,99$; RMSE $A=0,06)$. A LMX-MDM se associou positivamente à satisfação no trabalho $(r=0,11 \mathrm{a} 0,76 ; p<0,05)$ e às competências gerenciais $(r=0,60$ a 0,$80 ; p<0,01)$, e negativamente à intenção de rotatividade $(r=-0,33$ a $-0,36 ; p<0,01)$. A partir desses resultados, observa-se a adequação da LMX-MDM para mensurar a qualidade da relação líder-membro, a qual demonstra associação com outros fenômenos organizacionais.

Palavras-chave: liderança; análise fatorial; validade
\end{abstract}

\section{Multidimensional Measure of Leader-Member Exchange: Psychometric Properties of its Brazilian Version}

\begin{abstract}
This paper aimed to identify evidences of validity of the internal structure of the Brazilian version of the multidimensional Leader-Member Exchange scale (LMX-MDM), which was administered to 363 people. Exploratory and confirmatory factorial analysis and correlation analysis with other constructs were performed. The scale presented nine items distributed in three loworder factors grouped into a higher-order LMX factor: Professional Respect $(\alpha=0.93)$, Affect $(\alpha=0.92)$ and Loyalty $(\alpha=0.90)$, explaining $69.39 \%$ of the variance of the phenomenon. Adjustment indicators were satisfactory $\left(\chi^{2} / \mathrm{g} .1=2.20, \mathrm{GFI}=0.97\right.$, $\mathrm{CFI}=0.99$, RMSEA $=0.06$ ). LMX-MDM was positively associated to job satisfaction $(\mathrm{r}=0.11$ to $0.76, \mathrm{p}<0.05)$ and managerial skills ( $r=0.60$ to $0.80, p<0.01)$, and negatively to turnover intention $(r=-0.33$ to $-0.36, p<0.01)$. These findings suggest that the LMX-MDM presents evidences of validity for use in measuring quality of the leader-member relationship, which is associated to other organizational phenomena.

Keywords: leadership; factor analysis; validity
\end{abstract}

Calidad de Relación Líder-Miembro: Evidencias de Validez de Escala Multidimensional

\begin{abstract}
Resumen
El objetivo de este artículo fue identificar evidencias de validez de estructura interna de la versión brasileña de la escala multidimensional de calidad de relación líder-miembro (LMX-MDM). Se aplicó la LMX-MDM a 363 personas. Se realizó análisis factorial exploratorio y confirmatorio, y análisis de correlación con otros constructos. La escala presentó nueve ítems distribuidos en tres factores de primer orden que se agrupan en un factor global: Respeto Profesional $(\alpha=0,93)$, Afecto $(\alpha=0,92)$ y Lealtad $(\alpha=0,90)$ explicando 69,39\% de la varianza del fenómeno. Los indicadores de ajuste fueron satisfactorios $\left(\chi^{2} /\right.$ g.l. $=2,20$; GFI=0,97; CFI=0,99; RMSEA=0,06). La LMX-MDM se asoció positivamente a satisfacción en el trabajo ( $\mathrm{r}=0,11$ a 0,76 , $\mathrm{p}<0,05)$ y a las competencias gerenciales $(\mathrm{r}=0,60$ a $0,80, \mathrm{p}<0,01)$, y negativamente a la intención de rotatividad $(\mathrm{r}=-0,33$ a $-0,36$, $\mathrm{p}<0,01)$. A partir de estos resultados, se observa la adecuación de la LMX-MDM para medir la calidad de relación líder-miembro, la cual demuestra asociación con otros fenómenos organizacionales.

Palabras clave: liderazgo; análisis factorial; validez
\end{abstract}

\section{Introdução}

Têm sido crescentes as pesquisas sobre liderança, as quais têm incluído, em seus modelos, o líder, o liderado, o contexto, os níveis e suas interações dinâmicas. Dentre as diferentes abordagens de liderança, a teoria da troca líder-membro (Leader-Member Exchange - LMX) se destaca por tratar o processo de liderança tomando como foco de interesse a díade composta pelo líder e o membro da equipe, contrapondo-se às teorias tradicionais centradas apenas no líder (Day \& Miscenko, 2015; Yukl, 2009). Apesar de o início dos estudos sobre a LMX remontarem aos anos 70, a publicação sobre o tema é ainda rara no Brasil, conforme evidenciado 
na realização desta pesquisa. Além disso, as contribuições do estudo da LMX para a melhor compreensão do processo da liderança estão limitadas pelo fato de não contarmos com uma medida adequada disponível para avaliarmos a sua ocorrência. Tendo em vista esse cenário, a presente pesquisa tem por objetivo verificar as evidências de validade da estrutura interna de uma escala que avalia a qualidade da relação entre líderes e subordinados, a partir da teoria LMX.

Visando atingir o objetivo mencionado, este trabalho se inicia com a definição do construto de base, assim como a apresentação da lógica teórica em que a LMX se sustenta, uma vez que a mensuração de qualquer fenômeno somente pode ser bem sucedida a partir de uma delimitação teórica clara e precisa. $\mathrm{Na}$ perspectiva da LMX, a liderança é compreendida como um "processo de influência que leva as pessoas a compreenderem e concordarem sobre o que efetivamente precisa ser feito e como deve ser feito, além do processo de facilitação de esforços individuais e coletivos para a realização dos objetivos compartilhados" (Yukl, 2009). Assim, é tratada como um processo social, que envolve aspectos emocionais e racionais na interação entre ambos os atores envolvidos (líder e membro).

Considerando a liderança enquanto processo, o estudo da LMX surgiu a partir das teorias da tomada de papéis e das trocas sociais, conforme relatado por Graen e Uhl-Bien (1995) e por Liden e Maslyn (1998). Pontualmente, esses autores apontam que a maioria dos líderes desenvolve uma relação de alta qualidade com um pequeno número de subordinados, os quais atuam como assistentes ou conselheiros. Delegam a eles, então, tarefas mais interessantes e desejáveis, bem como maior responsabilidade, e também lhes são fornecidos mais recursos, atenção e oportunidades. Esses, em contrapartida, retribuem com melhor desempenho e lealdade.

A compreensão do construto da LMX sofreu alterações em sua natureza, como resultado de um processo de refinamento e evolução. Assim, a proposição teórica inicial foi desenvolvida por pesquisadores americanos na década de 1970 e inicialmente denominada de Ligação Diádica Vertical (VDL), tendo em vista o foco nos processos de influência recíproca compostos por alguém com autoridade sob outra pessoa, conforme relatam Day e Miscenko (2015). Esses autores ainda mencionam que a VDL surgiu em contraposição às teorias tradicionais de liderança, que consideravam como homogêneas as percepções dos membros do grupo, bem como os comportamentos dos supervisores em relação aos liderados. Ao colocar em questão a uniformidade dos estilos do líder, outro ponto de destaque da VDL era seu foco no processo de tomada de papéis. Assumia-se que o líder demandava um determinado papel ao membro da equipe, que o percebia e se comportava de determinada maneira, cuja resposta era recebida pelo líder como feedback. A partir da avaliação do comportamento do subordinado, a chefia poderia lhe atribuir novos papéis ou designá-los a outro membro.

Nos anos 80, o foco teórico mudou da VDL para a LMX, cuja diferença central é que a primeira enfatizava a noção de trocas diferenciadas, enquanto a LMX foca principalmente na resposta ao trabalho em função de variáveis relacionais, do próprio trabalho e suas interações, conforme apontam Graen e Uhl-Bien (1995). Assim, esses autores ressaltam que as proposições teóricas com foco nas trocas sociais assumiram um importante papel no desenvolvimento da LMX, já que a teoria visa, além da descrição de relacionamentos diferenciados, a explicação de como essas relações se desenvolvem e quais as consequências para a organização. Destacando as mudanças ocorridas na proposição teórica inicial da LMX, os autores apontam ainda que a essência da LMX passou a ser descrita da seguinte forma: 1) o desenvolvimento das relações é influenciado pelas características e comportamentos dos líderes e membros, mediante o processo de tomada de papéis e; 2) envolve relações de alta qualidade, com impacto positivo para os líderes, membros, as unidades de trabalho e a organização. Ou seja, assume-se que a liderança efetiva ocorre quando líderes e seguidores desenvolvem e mantêm uma troca social de alta qualidade. Tal compreensão da LMX permanece sendo utilizada atualmente, configurando-se, portanto, a base teórica adotada no presente estudo para se mensurar a qualidade da relação líder-membro.

Além de descrever a maneira como a LMX tem evoluído enquanto proposição teórica que explica a relação entre o líder e os liderados, é necessário discorrer quanto aos seus elementos constitutivos, tendo em vista que esses caracterizam o fenômeno, devendo, portanto, estar refletidos no instrumento que busca mensurá-lo. Ancorados na perspectivas teóricas que descrevem a LMX, os estudos empíricos têm subsidiado a noção de que a troca líder-membro é um construto multidimensional e deve ser medido como tal. Essas evidências, entretanto, não são convergentes no que diz respeito a quais são essas dimensões. Assim, os estudos iniciais (Dienesch \& Liden, 1986) propunham que a LMX era 
composta por afeto, contribuição e lealdade. Graen e Uhl-Bien (1995), entretanto, posteriormente sugeriram que a LMX era composta por respeito, confiança e obrigação. A argumentação desses autores ganha relevância, pois defendem adicionalmente que essas dimensões são tão altamente correlacionadas que podem ser agregadas em um único conjunto, sugerindo, portanto, uma estrutura unidimensional.

Em estudo posterior, foi proposto um modelo multidimensional da LMX, que incluía também confiança e respeito profissional (Liden \& Maslyn, 1998). A análise empírica da pertinência da proposta feita revelou, entretanto, a não discriminação entre as dimensões confiança e lealdade, razão pela qual os autores optaram pela retirada da dimensão acrescentada e pela redefinição da dimensão lealdade, de modo que passasse a refletir também noções de confiança. Assim, a versão final da escala proposta por esses autores foi composta por quatro fatores (Multidimensional Measure of Leader-Member Exchange - LMX-MDM): afeto, lealdade, contribuição e respeito profissional.

$\mathrm{O}$ afeto, segundo os autores, refere-se à afeição que o subordinado tem pelo seu chefe, que se baseia principalmente na atração interpessoal, em vez de trabalho ou de valores profissionais: uma relação de amizade, por exemplo. A lealdade é a percepção do subordinado de que o seu chefe expressa apoio público aos seus objetivos e ao seu caráter. Envolve fidelidade ao subordinado, geralmente de forma consistente de situação para situação. A contribuição, por outro lado, é o nível de esforço que o subordinado percebe colocar em atividades orientadas ao trabalho, em direção aos objetivos mútuos (explícitos ou implícitos) da dupla. É a medida em que o subordinado lida com responsabilidade e completa tarefas que vão além da descrição do trabalho. Por fim, o respeito profissional é a percepção do grau em que o chefe construiu uma reputação, dentro ou fora da organização, de excelência em sua linha de trabalho. Esta percepção pode ser baseada em dados históricos sobre a pessoa, comentários feitos por outros indivíduos, prêmios ou reconhecimento alcançado. Assim, é possível, embora não seja necessário, que o chefe tenha desenvolvido uma imagem de respeito profissional antes de o subordinado conhecê-lo ou trabalhar com ele.

Em uma revisão mais recente dos estudos sobre a LMX, foi verificado que a escala LMX-MDM pode ser modelada hierarquicamente, com quatro fatores de primeira ordem (afeto, lealdade, contribuição e respeito profissional) e um fator global de segunda ordem
(Joseph, Newman, \& Sin, 2011). No mesmo estudo é apontado que, apesar de vários pesquisadores evidenciarem a adequação empírica dos modelos de análise fatorial confirmatória hierárquica para a LMX-MDM, os estudiosos na área tendem a avaliá-la somente enquanto construto global, sem considerar suas dimensões.

Ao analisar a literatura sobre o tema, focando nas medidas adotadas, observa-se que, de fato, há importante lacuna de pesquisa ao examinar o LMX como construto multidimensional. Nota-se que a maioria dos estudos tem utilizado a escala unidimensional LMX-7 desenvolvida por Graen (Pellegrini, 2015), composta por sete itens e cujo uso tem sido questionado, dentre outros fatores, porque os itens apresentam opções de respostas distintas entre si, o que pode interferir nas análises (Yukl, 2009).

A conceituação multidimensional da LMX indica que diferentes domínios podem levar a diferentes resultados (Liden \& Maslyn, 1998; Pellegrini, 2015), sobretudo quando se consideram as características de diferentes culturas. De maneira pontual, Pellegrini sugere, a este respeito, que as quatro dimensões LMX-MDM sejam teórica e empiricamente significativas em todas as culturas. No entanto, pondera-se que, em relação às dimensões, o que é interpretado como comportamento positivo pode não ter uma conotação similar em outra cultura, fato que exige a análise cuidadosa do comportamento da medida ao ser aplicada em um novo contexto cultural.

Analisando a produção nacional sobre essa temática, observa-se que, no Brasil, o único estudo que adotou a LMX-MDM foi a tese de doutorado de Amaral (2007), quem traduziu e adaptou a escala à língua portuguesa. Nessa adaptação, entretanto, foram incluídos, por meio de revisão teórica, novos itens concebidos pelo próprio autor, a partir dos pressupostos das Teorias da Identidade Social e da Autocategorização, sem análise do construto por métodos qualitativos de pesquisa. Ressalta-se ainda que ambas as teorias não representam as bases teóricas utilizadas no desenvolvimento inicial da LMX e tampouco são observados estudos internacionais que adotem tal abordagem na avaliação do fenômeno.

Buscando obter evidências mais robustas da relação estabelecida entre a LMX e comportamentos organizacionais, foi realizada, no presente estudo, busca por artigos do tipo meta-análises em diferentes plataformas (ProQuest, Google Acadêmico, Periódicos CAPES, Scielo e Pubmed). Para tanto, foram adotados termos referentes à LMX e meta-análises. A ênfase dada 
a estudos desse tipo justifica-se pelo fato de trazerem estimativas robustas que, ao resultar da congregação de dados de diversas pesquisas e inúmeras amostras, revelam padrões de associação menos vulneráveis à especificidade dos estudos quando analisados de forma individualizada. Foram encontrados 12 estudos desse tipo, publicados entre 1997 e 2016, em que é demonstrada a existência de relação positiva entre a LMX e comportamentos de cidadania organizacional, desempenho, justiça, satisfação no trabalho, satisfação com a chefia, comprometimento organizacional, clareza de papéis e competências dos membros (Dulebohn, Bommer, Liden, Brouer, \& Ferris, 2012; Gerstner \& Day, 1997). Também se observa relação negativa entre a LMX e a intenção de rotatividade e a rotatividade real dos membros, sendo que quanto melhor a relação, menor o interesse dos subordinados em sair da empresa (Dulebohn et al., 2012; Rockstuhl, Dulebohn, Ang, \& Shore, 2012).

Ainda em relação aos correlatos da LMX, busca similar foi realizada na produção nacional (nas bases Scielo, ProQuest, Google Acadêmico, Periódicos CAPES, Pubmed, Banco de Teses e Dissertações da CAPES), sem se restringir, entretanto, a estudos do tipo meta-análise. Os resultados revelam defasagem se comparada à produção internacional, pois foram identificados apenas cinco artigos empíricos (Niemeyer \& Cavazotte, 2016; Nunes \& Gaspar, 2016; Oliveira \& Rocha, 2017; Oliveira \& Silva, 2015; Silveira \& Hanashiro, 2009) e um artigo de revisão (Turano \& Cavazotte, 2016). Também foram encontradas onze dissertações de mestrado e três teses de doutorado que adotaram a teoria (Amaral, 2007; Azevedo, 2014; Braga, 2012; Brant, 2012; D’Ávila, 2016; Furtado, 2016; Gonçalves, 2015; Horta, 2012; Maccarielo, 2012; Niemeyer, 2013; Pereira, 2016; Rocha, 2014; Silva, 2014; Silveira, 2007). Os estudos nacionais, de forma consistente com a literatura internacional, apontaram para a associação entre a LMX e variáveis que medem satisfação (Brant, 2012; Maccarielo, 2012; Nunes \& Gaspar, 2016; Silveira \& Hanashiro, 2009), engajamento no trabalho (Oliveira \& Rocha, 2017; Oliveira \& Silva, 2015; Rocha, 2014), comprometimento organizacional (D'Ávila, 2016), liderança ética (Niemeyer, 2013; Niemeyer \& Cavazotte, 2016) e liderança autêntica (Braga, 2012).

Diante dos benefícios decorrentes da manutenção de relações de alta qualidade entre o líder e o liderado, e tendo em vista a defasagem de estudos nacionais sobre a teoria LMX, trata-se de um momento oportuno para o Brasil (Turano \& Cavazotte, 2016), pois existe uma necessidade significativa de estudos da América Latina e outras regiões, para obter uma compreensão mais profunda do que impulsiona a qualidade LMX (Dulebohn et al., 2012; Pellegrini, 2015). Adicionalmente, são necessários estudos que a avaliem de maneira multidimensional, uma vez que as prioridades atribuídas a diferentes dimensões do LMX podem diferir entre as culturas. Assim, foi desenvolvido um estudo empírico com o objetivo de investigar as evidências de validade da estrutura interna de uma medida que avalia a qualidade da relação entre líderes e subordinados, a partir da teoria LMX.

\section{Método}

\section{Procedimentos}

Para atingir o objetivo de pesquisa, adotou-se como ponto de partida a Escala LMX-MDM, desenvolvida por Liden e Maslyn (1998), traduzida e adaptada por Amaral (2007). Isso porque foram utilizados apenas os itens que constam na escala LMX-MDM original, a qual conta com quatro fatores com três itens cada: Respeito Profissional, Afeto, Lealdade e Contribuição. A escala de resposta da medida era do tipo Likert de sete pontos $(1=$ discordo totalmente, $7=$ concordo totalmente). Considerando que os 11 itens originais da escala já haviam sido traduzidos (Amaral, 2007), foi realizado o procedimento de tradução-retrotradução português-inglês apenas para o item incluído posteriormente pelos próprios autores à escala original, cuja tradução final foi "Eu não me importo de trabalhar o meu máximo para o meu chefe". Ainda assim, a pertinência da redação em língua portuguesa foi analisada por um grupo de avaliadores. Adicionalmente, seis juízes foram informados dos conceitos das quatro dimensões e, em seguida, indicaram à qual dimensão cada item pertencia. Todos classificaram corretamente os itens nas respectivas dimensões. Superada essa fase, procedeu-se à aplicação da escala LMX-MDM, de modo a possibilitar a realização das análises compatíveis com o objetivo deste estudo. Destaca-se que, em todas as fases da pesquisa, as pessoas foram informadas do caráter voluntário da sua participação, com garantia do anonimato e sigilo das informações. Assim, respeitaram-se os princípios éticos que regem as pesquisas com seres humanos.

\section{Amostra}

A amostra foi composta por servidores e colaboradores de organizações públicas que participavam de cursos em uma escola de governo em Brasília/ 
DF entre junho e julho de 2017, por conveniência. Mediante autorização do diretor da escola, um dos pesquisadores entrou nas salas de aula, explicou os objetivos, garantiu o anonimato dos participantes e convidou-os a preencherem um questionário impresso e autoaplicado, o qual foi coletado posteriormente. A amostra final totalizou 363 participantes, cuja média de idade era 39,13 (DP: 9,45) e a maioria era homem $(52,6 \%)$. A média aritmética de tempo de trabalho na organização foi de 8,33 anos (DP:8,80), 94,5\% eram servidores ou empregados públicos, e a grande maioria atuava em instituições do poder executivo $(86,2 \%)$. Quanto à escolaridade do grupo, a maior parte tinha algum curso de especialização (36,1\%), seguido pelo grupo com graduação $(16,0 \%)$ ou mestrado completo $(15,4 \%)$. Graus educacionais acima e abaixo dos ora relatados apareceram em porcentagens inferiores a $5 \%$. Em relação à chefia, os respondentes relataram trabalhar, em média a 2,11 anos (DP: 2,74) sob a mesma chefia, e a maior parte estava sob comando de uma pessoa do sexo masculino $(61,2 \%)$.

\section{Instrumentos}

Além da LMX-MDM, foram coletados também os seguintes dados: sexo, idade, escolaridade e dados funcionais (tipo de instituição, vínculo institucional, tempo de trabalho na instituição, tempo de trabalho como subordinado à chefia e sexo da chefia). Para validação externa, também foram aplicadas as seguintes escalas:

Escala de avaliação das competências gerenciais: Trata-se de medida unifatorial composta por 30 itens. Desenvolvida para avaliar a atuação gerencial, foca nas competências dos gestores (Silva, Laros, \& Mourão, 2007) e foi respondida em escala do tipo Likert de 11 pontos (de "Concordo totalmente" a "Discordo Totalmente"). No presente estudo, a escala revelou estrutura unidimensional em análise fatorial exploratória. Três itens foram excluídos por apresentarem cargas fatoriais pobres e compartilhadas em mais de uma faceta. A estrutura mais satisfatória contemplou um fator e três facetas: Coordenação do trabalho (cinco itens; $\alpha=$ $0,95 ; r$ item total média $=0,86)$; Relações Interpessoais (seis itens; $\alpha=0,95 ; r$ item total média $=0,87$ ); e Visão, aprendizagem, inovação e comunicação (16 itens; $\alpha=$ $0,98 ; r$ item total média $=0,84)$.

Escala de satisfação no trabalho: A medida adotada (Coelho Junior \& Faiad, 2012) é composta por 24 itens e aborda as dimensões: satisfação com a chefia, os colegas, a natureza do trabalho, as promoções e o salário. As perguntas foram respondidas em escala de sete pontos, do tipo Likert (totalmente insatisfeito a totalmente satisfeito). Na presente amostra, todos os índices psicométricos foram satisfatórios: satisfação com a chefia $(\mathrm{S} 1 ; \alpha=0,93 ; r$ item total média $=0,82)$, os colegas $(\mathrm{S} 2$; $\alpha=0,88 ; r$ item total média $=0,73)$, as tarefas $(\mathrm{S} 3 ; \alpha=$ $0,86 ; r$ item total média $=0,71)$; as promoções $(\mathrm{S} 4 ; \alpha=$ $0,92 ; r$ item total $=0,79)$, e o salário $(\mathrm{S} 5 ; \alpha=0,93 ; r$ item total $=0,83)$.

Escala de intenção de rotatividade: Foi avaliada por medida unidimensional de três itens, adaptada de Siqueira, Gomide Júnior, Oliveira, e Polizzi Filho (2014). A escala original $(\alpha=0,95)$ apresenta itens relacionados a pensamentos, vontades e ações dirigidas à intenção de sair da empresa. $\mathrm{Na}$ presente pesquisa, o termo "empresa" foi alterado para "equipe", em função do fenômeno pesquisado, havendo índices adequados de confiabilidade $(\alpha=0,92 ; r$ item total média $=0,83)$. A escala de resposta era de frequência com cinco pontos, ancorada nas extremidades "Nunca" e "Sempre".

\section{Análise dos dados}

Inicialmente, foi realizada análise dos valores ausentes e extremos, bem como análise dos pressupostos estatísticos. Em seguida, foi realizada $A F E$, incluindo fatorabilidade, extração de fatores pelo método dos eixos principais, com rotação Promax, e análise de confiabilidade. Foi realizada ainda a análise fatorial confirmatória $(A F C)$, estimada pelo método da máxima verossimilhança. $\mathrm{Na} A F C$, foram testados dois modelos: 1) um modelo com os fatores específicos de primeira ordem, com um fator global de segunda ordem; e 2) um modelo unifatorial, com todos os itens em um único fator. Foram comparados os índices de ajuste e resíduos. Em seguida, buscou-se estabelecer associações entre a LMX-MDM e outros construtos, por meio da correlação de Pearson. Utilizou-se o software SPSS versão 23 e IBM Amos 23.

\section{Resultados}

Com o objetivo de proceder à investigação das evidências de validade da escala, verificou-se a ocorrência de erros de digitação e dados ausentes, assim como a análise de pressupostos estatísticos. Como a quantidade de casos omissos não ultrapassou $3 \%$ por item, optou-se pela exclusão pairwise dos valores ausentes. A partir da distância de Mahalanobis, dezesseis casos extremos multivariados foram identificados. As análises subsequentes foram realizadas com e sem esses outliers, não havendo diferenças nos resultados. Assim, optou-se 
por apresentar os dados obtidos com a amostra total, composta por 363 participantes. A normalidade da distribuição dos dados foi verificada mediante a inspeção dos valores de skeenness para cada item. Sucintamente, em relação à assimetria, é possível afirmar que nenhum valor ficou acima de dois (em valores absolutos) indicando não haver problema relevante de normalidade univariada, segundo os critérios defendidos por Miles e Shevlin (2001).

Para análise das evidências da validade psicométrica da escala LMX-MDM, em um primeiro momento, investigou-se a fatorabilidade da matriz de dados. Os resultados foram satisfatórios, tendo encontrado um determinante de baixa magnitude e diferente de zero e $K M O$ de 0,93 . A inspeção da matriz de correlações também sinalizou a pertinência de reduzir os itens a fatores, sendo todas significativas à exceção de um item que mostrou associação com alguns dos outros itens da medida, mas não com todos. Assim, excetuando-se esse item, as demais correlações bivariadas foram todas significativas e oscilaram entre 0,48 e 0,85 .

Após essa etapa, buscou-se definir o número adequado de fatores a extrair da matriz de dados, adotando-se critérios teóricos e psicométricos. A natureza teórica do construto aponta estar composto por quatro dimensões: respeito profissional, afeto, lealdade e contribuição. Ainda assim, tendo em vista a sua natureza, não se espera independência entre eles, entendendo-se que se trata de componentes fortemente interligados de um mesmo construto. Além de observar critérios de pertinência teórica, foram adotados o gráfico do scree e o critério de Kaiser para definir o número adequado de fatores, os quais apontaram a pertinência de extrair até dois fatores, com variância explicada de 69,93\%. No primeiro fator, permaneceram os itens orginalmente descritos como Respeito Profissional. Todos os demais se mantiveram no segundo fator. Um dos itens referentes à Contribuição ("Faço tarefas para meu chefe que vão além das minhas atribuições"), entretanto, trouxe a carga mais baixa à solução fatorial $(0,39)$. Dessa forma, optou-se por retirar da análise os três itens de Contribuição e refazer nova extração de fatores, mantendo-se os itens referentes aos demais componentes. Com a retirada dos três itens, tanto na análise do scree plot, quanto pelo critério de Kaiser, a solução apontou para a extração de um fator, com variância explicada de $69,39 \%$ e cargas fatoriais entre 0,76 e 0,87 . A confiabilidade interna do fator com os nove itens se mostrou adequada ( $\alpha=0,95$ e $r$ item-total média de 0,78$)$.
Em seguida, a partir de critérios teóricos e psicométricos, optou-se por avaliar a existência de facetas de um mesmo fator, em função dos achados na literatura internacional identificarem na LMX os componentes Afeto, Respeito, Lealdade e Contribuição. Dessa forma, excluindo-se os itens de Contribuição, forçou-se a extração de três fatores com os itens restantes. Assim, a escala se comportou tal como proposto na literatura internacional (Tabela 1), sendo composta por Respeito Profissional (cargas fatoriais entre 0,82 e $0,93, \alpha=0,93$ e $r$ item-total média $=0,86$ ), Afeto (cargas entre 0,82 e $0,87, \alpha=0,92$ e $r$ item-total média $=0,84)$ e Lealdade (cargas entre 0,74 e $0,85, \alpha=0,90$ e $r$ item-total média $=0,80)$.

Em seguida, foi realizada análise confirmatória, retirando-se todos os casos que tinham dados ausentes. No modelo principal, com os três fatores de primeira ordem (Respeito Profissional, Afeto e Lealdade) agrupados no fator LMX global, os resultados demonstraram a adequação da medida: $\chi^{2} /$ g.l. $=2,20 ; G F I=0,97$; $A G F I=0,94 ; C F I=0,99 ;$ RMSE $A=0,059(I C 90 \%=$ 0,04-0,08). Os índices de ajustes foram todos acima de 0,90 e o intervalo de confiança a 90\% do RMSEA ficou ente 0,04 e 0,08 , indicando aceitável erro de aproximação (Thompson, 2000). Por outro lado, embora o $\chi^{2} /$ g.l. tenha sido superior a 1,96 , ressalta-se que o referido teste é sensível ao tamanho da amostra, levando à rejeição do modelo no caso de amostras grandes (Byrne, 2010). Assim, considerou-se o resultado satisfatório. Ademais, realizou-se a análise fatorial confirmatória de modelo concorrente para a versão unifatorial, com os nove itens sem separação por fatores, resultando em índices pouco satisfatórios: $\chi^{2} /$ g.l. $=19,69 ; G F I=0,70$; $A G F I=0,50 ; C F I=0,83$; RMSE $A=0,23$ (IC90\% $=0,22$ 0,25). Assim, para as análises subsequentes, foi adotado apenas o primeiro modelo testado na AFC, o qual considera a LMX como fenômeno multidimensional de segunda ordem.

Em seguida, realizou-se a verificação de validade externa, a partir da análise de correlação entre LMX e competências gerenciais, satisfação no trabalho e intenção de rotatividade (Tabela 2). Os resultados encontrados evidenciaram associação entre todas as variáveis de satisfação no trabalho, sendo a relação mais intensa a ocorrida entre os três fatores da LMX e a satisfação com a chefia (LMX-A: $r=0,80$; LMX-R: $r=0,77$; LMX-L: $r=0,74)$. Na análise de correlação entre a LMX e competências gerenciais, também houve associação entre todas as variáveis. Neste caso, a relação mais forte ocorreu entre o Afeto (LMX-A) e as Relações 
Tabela 1.

Cargas fatoriais, correlação item-total e indice de confiabilidade dos fatores da LMX na análise fatorial exploratória

\begin{tabular}{|c|c|c|c|c|}
\hline Fatores & Itens & \multicolumn{2}{|c|}{ Cargas fatoriais } & $\begin{array}{l}\text { Correlação } \\
\text { item-total }\end{array}$ \\
\hline \multirow{3}{*}{$\begin{array}{l}\text { Respeito } \\
\text { profissional } \\
(\alpha: 0,93)\end{array}$} & $\begin{array}{l}\text { Eu me impressiono com o conhecimento do meu chefe } \\
\text { sobre seu trabalho. }\end{array}$ & 0,93 & & 0,86 \\
\hline & Eu admiro as habilidades profissionais do meu chefe. & 0,83 & & 0,88 \\
\hline & $\begin{array}{l}\text { Eu respeito o conhecimento do meu chefe e sua } \\
\text { competência no trabalho. }\end{array}$ & 0,82 & & 0,84 \\
\hline \multirow{3}{*}{$\begin{array}{l}\text { Afeto } \\
(\alpha: 0,92)\end{array}$} & Meu chefe é uma pessoa com a qual é agradável trabalhar. & 0,87 & & 0,86 \\
\hline & $\begin{array}{l}\text { Meu chefe é o tipo de pessoa que qualquer um gostaria de } \\
\text { ter como amigo. }\end{array}$ & 0,86 & & 0,85 \\
\hline & Eu gosto muito do meu chefe como pessoa & 0,82 & & 0,83 \\
\hline \multirow[t]{3}{*}{$\begin{array}{l}\text { Lealdade } \\
(\alpha: 0,90)\end{array}$} & $\begin{array}{l}\text { O meu chefe me defende no trabalho perante seus } \\
\text { superiores, mesmo sem o conhecimento de todas as questões } \\
\text { envolvidas. }\end{array}$ & & 0,85 & 0,78 \\
\hline & $\begin{array}{l}\text { Meu chefe me defenderia perante outros na organização se } \\
\text { eu cometesse um erro sem ter tido a intenção. }\end{array}$ & & 0,79 & 0,81 \\
\hline & $\begin{array}{l}\text { Meu chefe me defenderia se eu fosse "atacado(a)" no } \\
\text { trabalho }\end{array}$ & & 0,74 & 0,80 \\
\hline
\end{tabular}

Alfa de Cronbach unifatorial: 0,95

Variância total explicada: 69,39\%

Tabela 2.

Correlação de Pearson entre LMX, satisfação no trabalho, intenção de rotatividade e competências gerenciais

\begin{tabular}{|c|c|c|c|c|c|c|c|c|c|c|c|}
\hline & LMX-A & LMX-R & LMX-L & S1 & S2 & S3 & S4 & S5 & IR & C1 & $\mathrm{C} 2$ \\
\hline LMX-A & - & & & & & & & & & & \\
\hline LMX-R &, $70^{* *}$ & - & & & & & & & & & \\
\hline LMX-L &, $74^{*}$ &, $71^{* *}$ & - & & & & & & & & \\
\hline S1 &, $80^{* *}$ &, $77^{* *}$ &, $74^{* *}$ & - & & & & & & & \\
\hline S2 &, $27^{* *}$ &, $28^{* *}$ &, $32^{* *}$ &, $49^{* *}$ & - & & & & & & \\
\hline S3 & $26^{* *}$ &, $33^{* *}$ &, $30^{* *}$ &, $49^{* *}$ &, $49^{* *}$ & - & & & & & \\
\hline S4 &, $20^{* *}$ &, $27^{* *}$ &, $29^{* *}$ &, $42^{* *}$ &, $38^{* *}$ &, $53^{* *}$ & - & & & & \\
\hline S5 &, $11^{*}$ &, $12^{*}$ &, $17^{* *}$ &, $24^{* *}$ &, $31^{* *}$ &, $31^{* *}$ &, $57^{* *}$ & - & & & \\
\hline IR &,$- 33^{* *}$ &,$- 36^{* *}$ &,$- 34^{* *}$ &,$- 47^{* *}$ &,$- 27^{* *}$ &,$- 56^{* *}$ &,$- 33^{* *}$ &,$- 17^{* *}$ & - & & \\
\hline C1 &, $63^{* *}$ &, $70^{* *}$ &, $60^{* *}$ &, $76^{* *}$ &, $37^{* *}$ &, $35^{* *}$ &, $33^{* *}$ &, $16^{* *}$ &,$- 38^{* *}$ & - & \\
\hline $\mathrm{C} 2$ &, $80^{* *}$ &, $69^{* *}$ &, $70^{* *}$ &, $81^{* *}$ &, $32^{* *}$ &, $32^{* *}$ &, $34^{* *}$ &, $21^{* *}$ &,$- 42^{* *}$ &, $83^{* *}$ & - \\
\hline C3 &, $70^{* *}$ &, $76^{* *}$ &, $69^{* *}$ &, $83^{* *}$ &, $39^{* *}$ &, $40^{* *}$ &, $38^{* *}$ &, $21^{* *}$ &,$- 44^{* *}$ &, $89^{* *}$ & ,90** \\
\hline
\end{tabular}

$* p<0,05 ; * * p<0,01$

LMX-A = LMX - Afeto; LMX-R = LMX - Respeito profissional; LMX-L = LMX - Lealdade; S1 = satisfação com a chefia; S2 = satisfação com os colegas; S3 = satisfação com a natureza do trabalho; S4 = satisfação com as promoções; S5 = satisfação com o salário; IR = intenção de rotatividade (intenção de sair da equipe); C1 = Competências - Coordenação do trabalho; C2 = Relações Interpessoais; C3 = Competências - Visão, aprendizagem, inovação e comunicação 
Interpessoais (C2: $\mathrm{r}=0,80)$. Também foi elevada a associação entre o Respeito Profissional (LMX-R) e as competências voltadas à visão, aprendizagem, inovação e comunicação (C3: $r=0,75)$. Por fim, a intenção de sair da equipe apresentou associação inversamente proporcional com todos os fatores da LMX (LMX-A: $r=$ -0,33; LMX-R: $r=-0,36$; LMX-L: $r=-0,34)$.

\section{Discussão}

A presente pesquisa tinha como objetivo investigar as evidências de validade da estrutura interna da versão brasileira da escala multidimensional da qualidade da relação líder-membro (LMX-MDM). $\mathrm{Na}$ amostra deste estudo, a LMX comportou-se ligeiramente diferente do que vem sendo observado em pesquisas internacionais, mas com bons índices psicométricos. Embora os autores da escala original (Liden \& Maslyn, 1998) tenham proposto uma escala multidimensional com quatro fatores (Respeito profissional, Afeto, Lealdade e Contribuição), a escala final, na presente amostra, apresentou apenas três fatores de primeira ordem (Respeito Profissional, Afeto e Lealdade). Ainda assim, os índices alcançados na análise fatorial confirmatória para a estrutura com os três fatores (e um global, de segunda ordem) foram satisfatórios e bem melhores se comparados aos obtidos na solução unifatorial. Ou seja, os resultados apontam que as decisões tomadas nos diversos momentos da pesquisa foram adequadas e eficazes, uma vez que a medida resultante do estudo realizado se constitui em ferramenta confiável para mensuração da qualidade das relações mantidas entre o líder e os membros da equipe.

As análises realizadas no presente estudo demonstraram a pertinência de se adotar uma solução fatorial que condensa três dimensões relacionadas, todas as quais se associam a um fator de segunda ordem. Com relação a esse arranjo, cabe apontar, em primeiro lugar, que os resultados da AFE e da AFC são coerentes entre si, já que ambos demonstraram a existência de um fator latente comum às dimensões em que os itens da medida se organizaram. Adicionalmente, ao se comparar os dois modelos testados na AFC, foi possível verificar a inadequabilidade de se adotar a escala LMX-MDM de modo unidimensional, sem a separação por fatores, tendo em vista os valores psicométricos pouco satisfatórios. Por fim, a correlação entre os três fatores (Afeto, Lealdade e Respeito Profissional) variou de 0,70 a 0,74, sugerindo que compartilham aproximadamente $49 \% \mathrm{da}$ variância, demonstrando suficiente especificidade entre si. Esse resultado coloca em questionamento a proposta de Graen e Uhl-Bien (1995), que sugerem que as dimensões da LMX são tão altamente correlacionadas que podem ser agrupadas em uma única medida. Considerá-la de modo unidimensional pode levar a perda de informações importantes sobre as relações de alta qualidade e suas associações com possíveis variáveis critério (Greguras \& Ford, 2006; Liden \& Maslyn, 1998).

Os resultados da presente pesquisa, então, corroboram os achados de estudos internacionais, os quais mencionam que as dimensões da LMX se agrupam em um construto de segunda ordem (Joseph et al., 2011; Liden, Wu, Cao, \& Wayne, 2016). Reforçam, portanto, o uso da escala LMX-MDM e a inclusão dos diferentes fatores nos modelos de pesquisa.

Em consonância com esse achado, embora na presente amostra os três fatores da LMX tenham se correlacionado com todas as variáveis estudadas, é importante destacar que a magnitude dessas correlações é diferente. Quando comparada a qualidade da relação com a satisfação com a chefia, por exemplo, a correlação foi maior para o Afeto (LMX-A: $r=0,80)$ e menor para a Lealdade (LMX-L: $r=0,74)$. Esse resultado vai ao encontro dos achados da literatura internacional, em que se sugere que a satisfação com a chefia aborda principalmente aspectos afetivos e interpessoais do relacionamento entre chefia e subordinado (Greguras $\&$ Ford, 2006).

Ainda em relação à satisfação no trabalho, a LMX se associou não somente com a satisfação com a chefia, mas também com a satisfação com os colegas, com a natureza do trabalho, com promoções e salário. Em outros estudos nacionais, também foram verificadas associações entre a LMX e a satisfação, embora tenham adotados instrumentos diferentes dos aqui utilizados (Brant, 2012; Maccarielo, 2012). A associação positiva entre a LMX e satisfação com o salário tem sido evidenciada em diversos estudos, cujos autores alegam que os subordinados em relações de alta qualidade apresentam atitudes mais positivas e, portanto, percebem menos discrepâncias em relação ao pagamento (Dulebohn et al., 2012). Por outro lado, não foram encontrados estudos que avaliassem a relação entre a LMX e a satisfação com os colegas, a natureza do trabalho e as promoções. Ainda assim, pode se argumentar que na existência de relações positivas com o chefe, maiores possibilidades de obter promoções se apresentam ao trabalhador, tendo em vista que usualmente os chefes são atores chamados a opinar em casos de avanço na carreira dos seus subordinados. 
Os resultados do presente estudo também apontam a existência de associações positivas, em consonância com a teoria proposta, porque além de favorecer a percepção de atitudes positivas (Dulebohn et al., 2012), a alta qualidade da relação propicia aos subordinados a atuação em tarefas mais interessantes e desejáveis, recebendo mais recursos, atenção e oportunidades.

Em relação às competências gerenciais, a relação mais forte ocorreu entre o Afeto (LMX-A) e a faceta de competências referente às Relações Interpessoais (LMX-A: $r=0,80$ ). Dessa forma, é importante destacar que o fator Afeto se refere, de fato, à afeição que o subordinado tem pelo seu chefe, fundamentada principalmente na atração interpessoal, em vez de trabalho ou de valores profissionais (Liden \& Maslyn, 1998). Sendo assim, era esperado que o Afeto se associasse mais fortemente às Relações Interpessoais, que corresponde à capacidade do líder em interagir e negociar, demonstrando respeito e assertividade (Silva et al., 2007). Por outro lado, a correlação da faceta de "Visão, aprendizagem, inovação e comunicação" foi maior com o Respeito Profissional (LMX-R: $r=0,75)$ se comparados ao Afeto e à Lealdade (LMX-A: $r=0,70$; LMX-L: $r=$ 69). Tal achado pode ser explicado pelo fato de o Respeito Profissional se referir à reputação de excelência do líder em sua linha de trabalho, cujos itens da escala remetam às competências das chefias. Assim, chefes que expressam competências voltadas ao desenvolvimento das equipes - visão, aprendizagem, inovação e comunicação - tendem a firmar relações de alta qualidade com seus subordinados.

A intenção de sair da equipe, por outro lado, associou-se negativamente com todos os fatores da LMX (LMX-A: $r=-0,33$; LMX-R: $r=-0,36$; LMX-L: $r=-0,34)$, com magnitudes semelhantes, corroborando achados internacionais (Dulebohn et al., 2012; Rockstuhl et al., 2012). Cabe ressaltar que, no presente estudo, avaliou-se a intenção de sair da equipe, em vez do interesse de sair da empresa. Tal escolha ocorreu principalmente em função da natureza do fenômeno estudado (que se associa inerentemente às equipes) $\mathrm{e}$ da amostra a ser pesquisada, partindo do pressuposto de que o interesse em sair da instituição geralmente reflete o interesse por buscar organizações com carreiras financeiramente mais atrativas. Sugere-se, por outro lado, que o interesse em sair da equipe esteja mais relacionado a aspectos que envolvam a liderança, a equipe e o trabalho exercido, o que se confirmou nas correlações encontradas.
Em relação ao fator de Contribuição da LMX, que não se sustentou no presente modelo, cabe destacar que os próprios autores da escala original apresentaram dificuldades para composição do fator. Dentre 30 itens propostos pelos autores, apenas dois se sustentaram na proposição inicial da escala, sendo necessária a retirada de itens que refletiam as percepções dos subordinados sobre os comportamentos dos líderes que favoreciam a contribuição dos seguidores (Liden \& Maslyn, 1998) e a inclusão de um novo item já ao final da pesquisa. $\mathrm{Na}$ literatura internacional, constatou-se que um estudo relata que, na análise de conteúdo dos itens da LMX-MDM por juízes (Joseph et al., 2011), a dimensão Contribuição parece avaliar o construto de comportamento de cidadania organizacional. Os mesmos autores destacam que, na presença de itens desse outro construto, os itens da dimensão Contribuição apresentam baixas cargas fatoriais.

Tal achado é inclusive similar aos dados obtidos na única pesquisa que adotou a LMX-MDM no Brasil (Amaral, 2007), em que os dois itens utilizados pra mensurar a dimensão da Contribuição apresentaram comunalidades bem abaixo do esperado na análise fatorial. Ou seja, os resultados em ambos os estudos apontam para a fragilidade dessa dimensão também no contexto da cultura brasileira. Ademais, especificamente em relação à amostra ora estudada, a grande maioria dos respondentes eram servidores públicos, cujas atribuições estão previstas em legislação. Assim, realizar atividades que vão além das atribuições do cargo ocupado, tal como proposto nos itens de Contribuição, poderia ser entendido como desvio de função. Dessa forma, é pertinente a constatação de que a dimensão Contribuição não tenha se sustentado na presente amostra, ao menos não da forma como apresentada na escala de Liden e Maslyn (1998).

Cabe destacar, entretanto, que representa uma limitação do presente artigo a amostra ser composta majoritariamente por servidores públicos. Assim, dada a ausência de pesquisas brasileiras sobre a LMX-MDM, recomenda-se que novos estudos incluam amostras de profissionais de empresas privadas brasileiras, de tal modo que seja possível verificar a estabilidade da solução fatorial em outras amostras. Ademais, apesar de não ter sido objetivo específico da presente pesquisa, sua natureza transversal não permite o estabelecimento de causalidade entre as variáveis. Trata-se, então, de outra limitação do estudo. Recomenda-se, portanto, a realização de pesquisas experimentais e longitudinais para 
melhor compreensão da relação entre a LMX e outras variáveis.

Apesar das limitações supracitadas, os resultados permitem concluir que os procedimentos adotados foram válidos e resultaram em uma escala de mensuração confiável. Sugere-se, portanto, a utilização da LMX-MDM em estudos sobre o tema no Brasil, de modo que se possa testar possíveis diferenças entre modelos de predição considerando seus três fatores de primeira ordem, ampliando a compreensão da influência desse construto em fenômenos organizacionais. Sugere-se que os três fatores sejam sempre incluídos, conjuntamente, nas ações de diagnóstico ou pesquisas sobre esta temática, pois essa estrutura possibilita obter informações que cobrem o espectro teórico do fenômeno definido como qualidade da relação líder-membro.

É, de fato, relevante a utilização da LMX-MDM no contexto brasileiro, porque a LMX se relaciona a diferentes construtos, tal como verificado na literatura internacional. Nota-se, portanto, que o presente estudo contribui consideravelmente para a literatura na área e para as práticas organizacionais. Do ponto de vista organizacional, é inegável a importância de se estabelecer boas relações entre chefia e subordinado, já que se associa positivamente à satisfação no trabalho e negativamente à intenção de rotatividade. Assim, estudos futuros podem contribuir ainda mais para o desenvolvimento organizacional ao considerar o impacto da LMX na efetividade das equipes e das organizações. Por fim, pesquisas futuras também devem favorecer a compreensão dos fatores que auxiliam no desenvolvimento e na melhoria da relação líder-membro, tais como as competências gerenciais. Os antecedentes da LMX apresentam-se como aspecto chave para explorar todo potencial da LMX, nos diferentes níveis organizacionais (Bauer \& Erdogan, 2015).

\section{Referências}

Amaral, D. J. (2007). Teoria da Troca entre Lider Liderado (LMX): Uma teoria diádica de liderança para a identifcação dos aspectos que compõem a interação líder-liderado. (Tese de doutorado), Universidade Presbiteriana Mackenzie, São Paulo. Recuperado de http://tede. mackenzie.br/jspui/handle/tede/841

Azevedo, E. M. A. (2014). Antecedentes da satisfação e do engajamento de funcionários de uma empresa pública. (Dissertação de mestrado não publicada), IBMEC, Rio de Janeiro.
Bauer, T. N., \& Erdogan, B. (2015). Leader-Member Exchange (LMX) Theory: An Introduction and Overview. In T. N. Bauer \& B. Erdogan (Eds.), The Oxford Handbook of Leader-Member Exchange. Oxford: Oxford University Press.

Braga, D. F. (2012). Liderança autêntica: Efeito sobre o desempenho e no comportamento de cidadania organizacional. (Dissertação de mestrado), IBMEC, Rio de Janeiro. Recuperado de https://www.ibmec.br/rj/ dissertacoes-de-mestrado-profissional-em-administracao-2012

Brant, P. S. (2012). A relação entre líderes e membros (LMX), estilos de liderança e suas influências na satisfação no trabalho. (Dissertação de mestrado), FUMEC, Belo Horizonte. Recuperado de http://www.fumec.br/ revistas/pdma/article/view/4500

Byrne, B. M. (2010). Structural equation modeling with Amos: basic concepts, applications, and programming (2 ed.). Ottawa: Routledge.

Coelho Junior, F. A., \& Faiad, C. (2012). Evidências de Validade da Escala de Satisfação no Trabalho. Avaliação Psicológica, 11(1), 111-121.

D’Ávila, P. R. (2016). Liderança, qualidade de troca social em equipes de trabalbo e o comprometimento organizacional: Um estudo com peacekeepers brasileiros no Haiti. (Tese de doutorado), Fundação Getúlio Vargas, Rio de Janeiro. Recuperado de http://bibliotecadigital. fgv.br/dspace/handle/10438/16721

Day, D. V., \& Miscenko, D. (2015). Leader-Member Exchange (LMX): Construct Evolution, Contributions, and Future Prospects for Advancing Leadership Theory. In T. N. Bauer \& B. Erdogan (Eds.), The Oxford Handbook of Leader-Member Exchange. Oxford: Oxford University Press.

Dienesch, R. M., \& Liden, R. C. (1986). Leader-Member Exchange Model of Leadership: A Critique and Further Development. The Academy of Management Review, 11(3), 618.

Dulebohn, J. H., Bommer, W. H., Liden, R. C., Brouer, R. L., \& Ferris, G. R. (2012). A Meta-Analysis of Antecedents and Consequences of Leader-Member Exchange: Integrating the Past with an Eye toward the Future. Journal of Management, 38(6), 1715-1759. doi: 10.1177/0149206311415280

Furtado, L. M. G. P. (2016). Leader-Member Exchange (LMX) within team contexts: a look Psico-USF, Bragança Paulista, v. 24, n. 3, p. 569-581, jul./set. 2019 
beyond the leader-member dyad. (Tese de doutorado), Fundação Getúlio Vargas, Rio de Janeiro. Recuperado de http://bibliotecadigital.fgv.br/dspace/ handle/10438/17797

Gerstner, C. R., \& Day, D. V. (1997). Meta-Analytic Review of Leader-Member Exchange Theory: Correlates and Construct Issues. Journal of Applied Psychology, 82(6), 827-844.

Gonçalves, R. C. (2015). Antecedentes do comportamento de retaliação: estudo em uma grande empresa brasileira de economia mista. (Dissertação de mestrado), IBMEC, Rio de Janeiro. Recuperado de Catálogo de Teses e Dissertações CAPES.

Graen, G. B., \& Uhl-Bien, M. (1995). Relationship-Based Approach to Leadership: Development of Leader-Member Exchange (LMX) Theory of Leadership over 25 Years: Applying a Multi-Level Multi-Domain Perspective. Leadership Quarterly, 6(2), 219-247.

Greguras, G. J., \& Ford, J. M. (2006). An examination of the multidimensionality of supervisor and subordinate perceptions of leader-member exchange. Journal of Occupational and Organizational Psychology, 79(3), 433-465.

Horta, J. R. (2012). Mulheres na Liderança: Efeitos do Gênero em avaliações de desempenho. (Dissetação de mestrado), Pontifícia Universidade Católica, Rio de Janeiro. Recuperado de https://www.maxwell. vrac.puc-rio.br/20778/20778_1.pdf

Joseph, D. L., Newman, D. A., \& Sin, H.-P. (2011). Leader-Member Exchange (LMX) Measurement: Evidence for Consensus, Construct Breadth, and Discriminant Validity. In D. D. Bergh \& D. J. Ketchen (Eds.), Research Methodology in Strategy and Management: Building Methodological Bridges (Vol. 6, pp. 89-135). Bingley, UK: Emerald Group Publishing Limited.

Liden, R. C., \& Maslyn, J. M. (1998). Multidimensionafity of Leader-Member Exchange: An Empirical Assessment through Scale Development. Journal of Management, 24(1), 43-72.

Liden, R. C., Wu, J., Cao, X., \& Wayne, S. J. (2016). Leader-Member Exchange Measurement. In T. N. Bauer \& B. Erdogan (Eds.), The Oxford Handbook of Leader-Member Exchange (pp. 29-54). Oxford: Oxford University Press.
Maccarielo, F. M. (2012). Liderança e satisfação no trabalho: uma visão dos seguidores. (Dissertação de mestrado), IBMEC, Rio de Janeiro. Recuperado de http://s3.amazonaws.com/public-cdn.ibmec. $\mathrm{br} /$ portalibmec-content/public/arquivos/df/ dis_2012_16_-_luis_felipe_younes_do_amaral. pdf

Miles, J., \& Shevlin, M. (2001). Applying Regression and Correlation: A Guide for Students and Researchers. Londres: Sage Publication.

Niemeyer, J. R. L. (2013). Ética na liderança e LMX (Leader Member Exchange): uma análise sobre o desempenho individual. (Dissertação de mestrado), Pontifícia Universidade Católica, Rio de Janeiro. Recuperado de https://www.maxwell.vrac.puc-rio. br/23907/23907.pdf

Niemeyer, J. R. L., \& Cavazotte, F. S. C. N. (2016). Ethical leadership, leader-follower relationship and performance: A study in a telecommunications company. Revista de Administração Mackenæie, 17(2), 67-92.

Nunes, E. M. G. T., \& Gaspar, M. F. M. (2016). A liderança em enfermagem e a satisfação dos pacientes em contexto hospitalar. Revista Gaúcha de Enfermagem, 35(2), e55726. doi: 10.1590/1983-1447.2016.02.55726

Oliveira, L. B., \& Rocha, J. C. (2017). Engajamento no trabalho: antecedentes individuais e situacionais e sua relação com a intenção de rotatividade. Revista Brasileira de Gestão de Negócios, 19(65), 415-431.

Oliveira, L. B., \& Silva, F. F. R. (2015). The Effects of High Performance Work Systems and Leader-Member Exchange Quality on Employee Engagement: Evidence from A Brazilian Non-Profit Organization. Procedia Computer Science, 55, 1023-1030. doi: 10.1016/j.procs.2015.07.092

Pellegrini, E. K. (2015). Relational leadership through the lens of international LMX research. In T. N. Bauer \& B. Erdogan (Eds.), The Oxford handbook of Leader-Member Exchange. Oxford: Oxford University Press.

Pereira, G. S. (2016). Liderando com bumildade: um estudo sobre o impacto da humildade do líder no desempenho e na cidadania dos liderados. (Dissertação de mestrado), Fundação Getúlio Vargas, Rio de Janeiro. Recuperado de http://bibliotecadigital.fgv.br/dspace/ handle/10438/17761 
Rocha, J. C. (2014). Antecedentes situacionais e individuais do engajamento no trabalho. (Dissertação de mestrado), IBMEC, Rio de Janeiro. Recuperado de http://s3.amazonaws.com/public-cdn.ibmec. $\mathrm{br} /$ portalibmec-content/public/arquivos/df/ dis_2014_32_-_juliana_da_costa_rocha.pdf

Rockstuhl, T., Dulebohn, J. H., Ang, S., \& Shore, L. M. (2012). Leader-member exchange (LMX) and culture: a meta-analysis of correlates of LMX across 23 countries. Journal of Applied Psychology, 97(6), 1097-1130. doi: 10.1037/a0029978

Silva, F. F. R. (2014). O impacto da gestão de recursos bumanos e da liderança sobre o engajamento: um estudo com empregados do Operador Nacional do Sistema Elétrico. (Dissertação de mestrado não publicada), IBMEC, Rio de Janeiro.

Silva, W., Laros, J. A., \& Mourão, L. (2007). Desenvolvimento e validação de escalas para avaliação da atuação gerencial. Revista Psicologia, Organizações e Trabalho, 7(1), 7-30.

Silveira, N. S. P. (2007). Similaridade e Dissimilaridade: A influencia da diversidade na qualidade da relação de superiores e subordinados. (Dissertação de mestrado em Administração), Universidade Presbiteriana Mackenzie, São Paulo. Recuperado de http://tede. mackenzie.br/jspui/handle/tede/565
Silveira, N. S. P., \& Hanashiro, D. M. M. (2009). Similaridade e Dissimilaridade entre Superiores e Subordinados e suas Implicações para a Qualidade da Relação Diádica. Revista de Administração Contemporânea, 13(1), 117-135.

Siqueira, M. M. M., Gomide Júnior, S., Oliveira, A. F., \& Polizzi Filho, A. (2014). Intenção de rotatividade. In M. M. M. Siqueira (Ed.), Novas medidas do comportamento organizacional: Ferramentas de diagnóstico e de gestão (pp. 209-216). Porto Alegre: Artmed.

Thompson, B. (2000). Ten commandments of structural equation modeling. In L. G. Grimm \& P. R. Yarnold (Eds.), Reading and Understanding More Multivariate Statistics (4 ed.). DC: American Psychological Association.

Turano, L. M., \& Cavazotte, F. (2016). Conhecimento Científico sobre Liderança: Uma Análise Bibliométrica do Acervo do The Leadership Quarterly. Revista de Administração Contemporânea, 20(4), 434-457.

Yukl, G. (2009). Leadership in Organizations (7 ed.). New Jersey: Pearson Education.

Recebido em: 25/06/2018

Reformulado em: 20/09/2018

Aprovado em: 05/10/2018 
Sobre as autoras:

Marcela Guimarães Côrtes é graduada em Fonoaudiologia (2007) e Mestre em Ciências da Saúde (2012) pela Universidade Federal de Minas Gerais. Atualmente é doutoranda em Psicologia Social, do Trabalho e das Organizações pela Universidade de Brasília. É Pesquisadora-Tecnologista em Informações e Avaliações Educacionais pelo Instituto Nacional de Estudos e Pesquisas Educacionais Anísio Teixeira, onde atua com Gestão de Pessoas.

ORCID: 0000-0003-3651-9798

E-mail: celacortes@gmail.com marcelag.cortes@inep.gov.br

Maira Gabriela Santos de Souza é graduada (2002) e Mestre (2006) em Psicologia pela Universidade de Brasilia. Atualmente é pesquisadora da Empresa Brasileira de Pesquisa Agropecuária. Tem experiência na área de Psicologia Organizacional e do Trabalho com ênfase em trabalho em equipe, treinamentos técnicos e gerenciais, levantamento de necessidades de treinamento, elaboração de diagnósticos organizacionais, construção e validação de escalas.

ORCID: 0000-0003-4211-9686

E-mail:souza.maira@gmail.com

Katia Elizabeth Puente-Palacios é graduada em Psicologia (1989) pela Ponificia Universidad Católica Del Ecuador, Mestre (1993) e Doutora (2002) em Psicologia pela Universidade de Brasília. Realizou pós-doutorado na Universidade de Valencia. Atualmente é professora associada na Universidade de Brasília. Sua área de expertise refere-se ao funcionamento de equipes de trabalho, utilizando prioritariamente métodos quantitativos, incluindo análise multinível, fatorial e regressão.

ORCID: 0000-0001-5598-8069

E-mail: kep.palacios@gmail.com

Contato com as autoras:

Coordenação-Geral de Gestão de Pessoas do Inep/MEC

SIG Quadra 04 lote 327 - 20 Andar

Brasília-DF, Brasil

CEP: 70610-908

Psico-USF, Bragança Paulista, v. 24, n. 3, p. 569-581, jul./set. 2019 